\title{
Research Paper \\ The Effect of Positive Psychology Intervention on the Psychological Well-Being of Adolescents
}

\author{
${ }^{*}$ Abolghasem Yaghoobi ${ }^{1}$ (), Bayan Nesai Moghadam² (1)
}

1. PhD. in Educational Psychology, Professor, Department of Psychology, Faculty of Economics and Social Sciences, Bu-Ali Sina University, Hamadan, Iran 2. PhD. Candidate, Department of Psychology, Faculty of Economics and Social Sciences, Bu-Ali Sina University, Hamadan, Iran.

\begin{tabular}{|c|c|}
\hline $\begin{array}{l}\text { Use yourd device to scan } \\
\text { and read the article online }\end{array}$ & Cftation Yaghoobi A, Nesai Moghadam B. [The Effect of Positive Psychology Intervention on the Psychological Well-Being \\
\hline Prita & $\begin{array}{l}\text { of Adolescents (Persian)]. Iranian Journal of Psychiatry and Clinical Psychology. 2019; 25(1):14-25. http://dx.doi.org/10.32598/ } \\
\text { ijpcp.25.1.14 }\end{array}$ \\
\hline 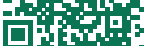 & dol http://dx.doi.org/10.32598/ijpcp.25.1.14 \\
\hline
\end{tabular}
Accepted: 01 Oct 2018 Available Online: 01 Apr 2019

Keywords: Positive psychology Psychological wellbeing, Adolescents

\begin{abstract}
A B S TRACT
Objectives The purpose of this research was to determine the effect of positive psychological well-being of adolescents.

Methods The research method was quais experimental. The statistical population of this study was all high school girl students in Serishabad of Kurdistan Province, Iran studying in the academic year of 19951996. The sample consisted of 30 high school female students who were randomly selected. Then they were randomly assigned to two groups: the experimental $(n=15)$ and control $(n=15)$ groups. The study tools used in this research was Ryff's Psychological Well-being Scale (RPWS). A pretest was administered to both the experimental and control groups. Then, the positive psychology intervention was administered to the experimental group, and the control group did not receive any intervention. At the end of the training, the post-test was taken for both groups.

Results The results of the Multivariate Analysis of Variance (MANOVA) indicate that positive psychology significantly increased psychological well-being among the experimental group compared to the control group. The results also showed a significant difference between the mean scores of RPWS of psychological well-being in the experimental and the control group in the post-test $(P<0.001)$. The positive psychology intervention method had a significant effect on autonomy, environmental mastery, personal growth, positive relation with others, purpose in life, and self-acceptance $(P<0.001)$.

Conclusion The interventional method of positive psychology can increase the psychological well-being of adolescents.
\end{abstract}

\section{Extended Abstract}

\section{Introduction}

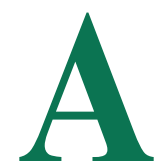

dolescence is a period of cognitive, biological, social, and emotional change [1]. It is also a critical period in cognitive, emotional, physical, and sexual cycle whose consequences can be seen in the future life of an individual [2]. Since adult behavior is established in adolescence, the influence of adolescence on the life of human beings and society is very significant [4]. Moreover, most mental health problems are formed in adolescence.

The results of epidemiological studies in the last two decades indicate that the first symptoms in $2 \%$ to $8 \%$ of depressed patients appear at the age of 16 . Other findings demonstrate that adolescents are more likely to develop severe depression,

* Corresponding Author:

Abolghasem Yaghoobi, PhD.

Address: Department of Psychology, Faculty of Economics and Social Sciences, Bu-Ali Sina University, Hamadan, Iran.

Tel: +98 (918) 1113987

E-mail: yaghobi41@yahoo.com 
anxiety, social dysfunction, academic failure, and even suicidal tendencies in the coming years [5]. Therefore, paying attention to the psychological well-being of adolescents is one of the strategies that may prevent these disorders. In recent years, the positivist approach of psychology has focused on human talents and abilities instead of dealing with abnormalities and disorders.

The ultimate goal of this approach is to identify the structures and practices that result in human happiness and wellbeing. Therefore, the most fundamental structures under study in this approach are the factors that make people more adaptive to the needs and threats of life [15]. There has been no research evidence on the effectiveness of the interventional methodology of positive psychology on the psychological well-being of adolescent girls in Serishabad area, Kurdistan Province. Regarding the importance of this issue in solving the problems of adolescent girls, this research attempted to investigate the effectiveness of the interventional method of positive psychology on the psychological well-being of adolescents.

\section{Method}

The present study was conducted at one of the girls' high schools in Serishabad, Kurdistan. The statistical population of this study comprised all female high schools students (aged 14-18 years) in Serishabad, Kurdistan, in the academic year of 2015-2016. One of the high schools in this region was se- lected using the available sampling method. Then, 30 samples were selected randomly among high school students who scored lower than the cut-off point in the test [22]. Then, they were randomly assigned to two groups of 15 subjects each.

The interventional method of positive psychology (based on the positive psychological intervention protocol on increasing the psychosocial well-being of young people from Jessica Sunshine University of South Florida, 2011) was performed on the experimental group [23]. The inclusion criteria were getting scores lower than the cut-off point in the Ryff's psychological well-being test, studying at secondary high school, being 14 to 18 years old, and not participating in other therapeutic programs. The exclusion criteria were being in the same grade twice or having a serious mental illness, a chronic medical condition, or organ failure.

Ryff's Psychological Well-being Scale (1989) was used as the study instrument. While the control group received no intervention, the positive-oriented psychology protocol, consisting of 10 sessions, each lasted one hour and a half(one session per week), was performed for the experimental group. At the end of the 10 sessions, the Ryff's psychological well-being questionnaire (the same as the pretest) was administered to the experimental and control groups.

\section{Results}

Tables 1 and 2 present the results of Multivariate Analysis of Variance (MANOVA) in the experimental and control groups

Table 1. Results of MANOVA

\begin{tabular}{ccccccc}
\hline Sources & Wilks' Lambda & df & F & Sig. & Eta $^{2}$ & Statistical Power \\
\hline Group & 0.051 & 2 & 253.127 & 0.001 & 0.949 & 100
\end{tabular}

The difference between the two groups regarding the positive intervention psychological method $\begin{aligned} & \text { Iranian Journal of } \\ & \text { PSYCHIATR AND CUNICAL PSYCHOLOGY }\end{aligned}$

Table 2. Summary of MANOVA on differences in terms of group membership in subscales of psychological well-being

\begin{tabular}{|c|c|c|c|c|c|c|c|}
\hline $\begin{array}{l}\text { Statistical Index } \\
\text { of Variables }\end{array}$ & $\begin{array}{c}\text { Total } \\
\text { Squares }\end{array}$ & df & Sig. & $\mathbf{F}$ & $\begin{array}{l}\text { Mean of } \\
\text { Squares }\end{array}$ & $\mathrm{Eta}^{2}$ & Statistical Power \\
\hline Self-governing & 19.544 & 1 & 0.001 & 245.858 & 19.544 & 0.898 & 1 \\
\hline $\begin{array}{l}\text { Dominate the } \\
\text { environment }\end{array}$ & 15.821 & 1 & 0.001 & 80.581 & 15.821 & 0.742 & 1 \\
\hline Personal growth & 16.875 & 1 & 0.001 & 49.327 & 16.875 & 0.638 & 1 \\
\hline $\begin{array}{l}\text { Positive relation- } \\
\text { ship with others }\end{array}$ & 16.449 & 1 & 0.001 & 153.804 & 16.449 & 0.846 & 1 \\
\hline $\begin{array}{l}\text { Having goals } \\
\text { in life }\end{array}$ & 13.621 & 1 & 0.001 & 32.240 & 13.621 & 0.535 & 1 \\
\hline Self-admission & 15.002 & 1 & 0.001 & 113.546 & 15.002 & 0.802 & 1 \\
\hline
\end{tabular}


regarding the psychological well-being and its subscales. According to the obtained results, there is a significant difference between the two groups considering the psychological well-being and its subscales $(\mathrm{P}<0.001)$. The students in the experimental group had higher performance on psychological well-being factors in the post-test than the control group. Therefore, the research hypothesis was confirmed. The Eta square was $95 \%$, i.e. $95 \%$ of the difference between the two groups is explained by the interventional method of positive-oriented psychology. The statistical significance of the test in the overall score and in all subscales is 1 , which indicates the adequacy of the sample size.

\section{Discussion}

In this study, the effectiveness of positive psychological intervention on adolescents' psychological well-being was studied. The data analysis indicated a difference between the adjusted post-test psychological well-being average scores in the experimental and control groups after the implementation of the positive psychological method. The results of this study showed the effectiveness of positive psychological approach for psychological well-being in adolescents. Furthermore, in this study, in order to control the potential biases, student sampling and placement in the control and experimental groups were carried out randomly. However, there were some limitations. First, the results can only be generalized to the females in the age range of 14-18 years and for other populations, the caution must be observed. Second, missing the longterm effect of this intervention is another limitation.

We suggest that the results of this research be used in medical institutions and secondary schools to help teenagers. Moreover, it is also recommended that organizations such as the Ministry of Education, the National Youth Organization, and the State Welfare Organization hold workshops for the development of mental health and positive behavior of the youth.

\section{Ethical Considerations}

\section{Compliance with ethical guidelines}

All ethical principles were considered in this article. The participants were informed about the purpose of the research and its implementation stages; they were also assured about the confidentiality of their information; Moreover, They were allowed to leave the study whenever they wish, and if desired, the results of the research would be available to them.

\section{Funding}

This research did not receive any specific grant from funding agencies in the public, commercial, or not-forprofit sectors.

\section{Authors contributions}

Conceptualization, data duration, analysis and drafting: Bayan Nesaimoghadam; Writing and review, Resources, and Data Curation: Abolqasem Yaghoubi.

\section{Conflict of interest}

The authors declared no conflict of interest. 


\title{
اثربخشى روش مداخلهاى روانشناسى مثبت ترا بر بهزيستى روانشناختى نوجوانان
}

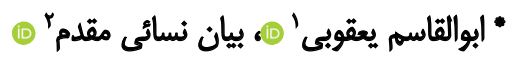 \\ ا- دكتراى روانشناسى تربيتى، استاد، كروه روانشناسى، دانشكده علوم اقتصادى واجتماعى، دانشعاه بوعلى سينا، همدان، ايران.

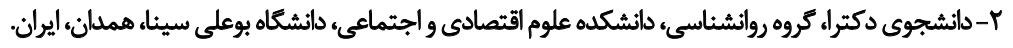

\begin{abstract}
حكبد
اهلهاف إين يُؤوهش با هدف بررسى اثربخشى روش مداخلهاى روانشناسى مثبتكرا بر بهزيستى روانشناختى نوجوانان اجرا شد.

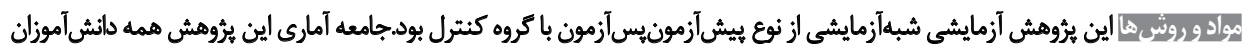

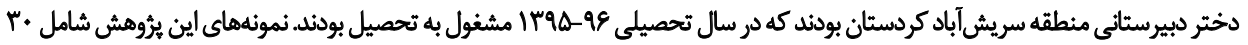

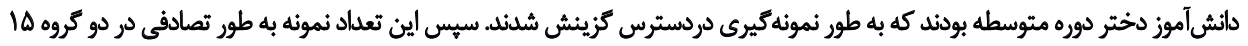

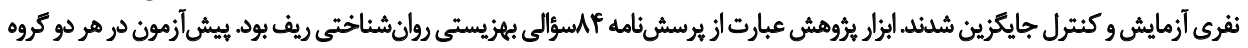

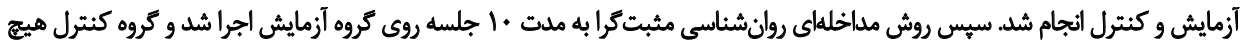

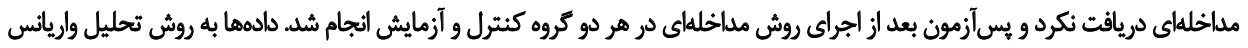
جئدمثغيرهتحليل شدند.

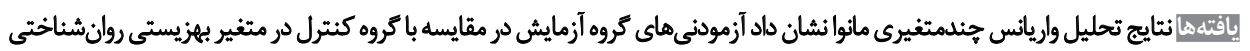

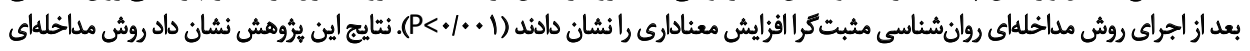

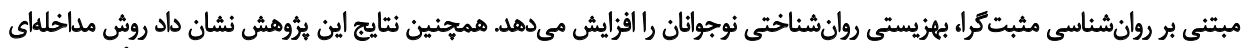

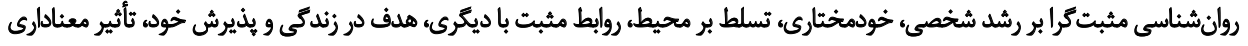

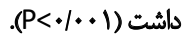

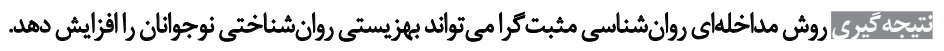

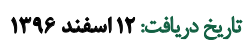

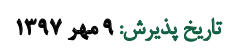

تاريخ انتشار: Tا فروردين

[f] و از سوى ديكر بيشتر مشكلات سلامت روان در سنين

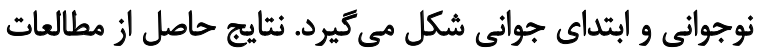

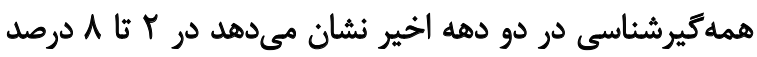

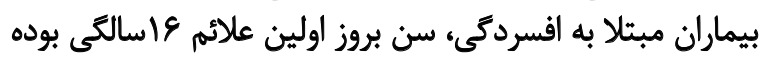

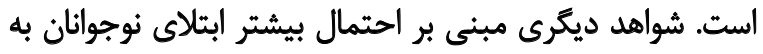

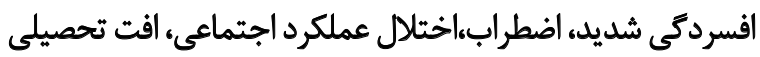

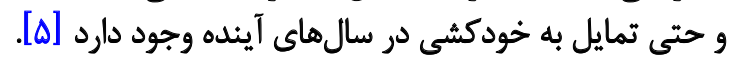

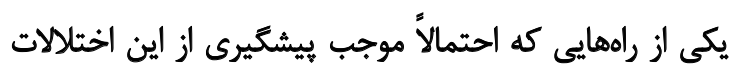

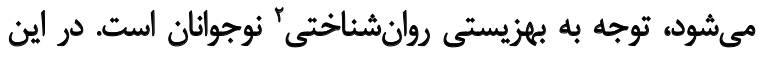

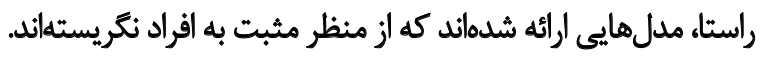

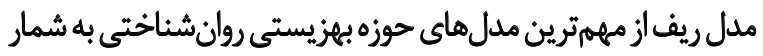

2. Psychological Well-Being (PWB) مقلمه

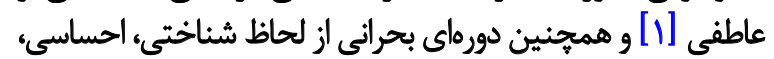

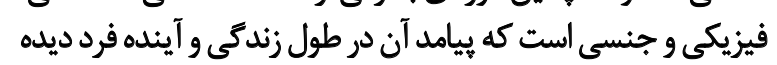

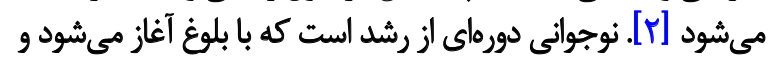

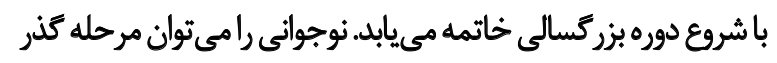

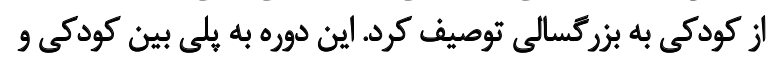

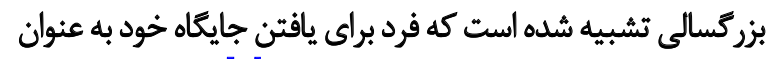

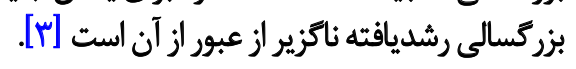

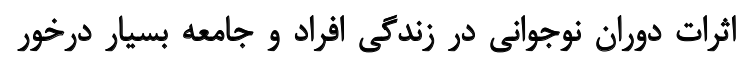

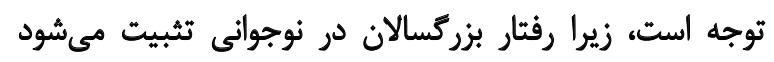

\section{Adolescence}


اخير از سوى روانشناسان توجه شده است اين رويكرد، هدف نهايى

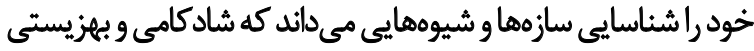

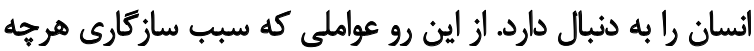

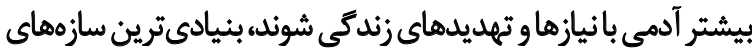

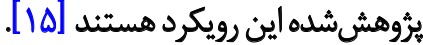
فرومن" معتقد است هيجانات مثبت، خصوصيات فكرى و رفتارى

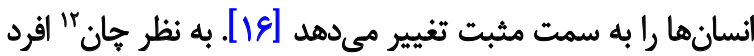

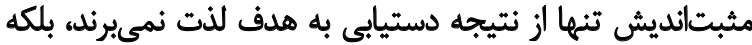

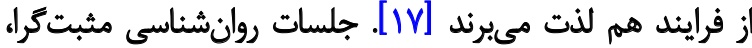

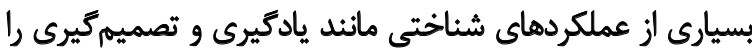

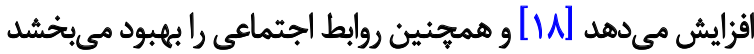

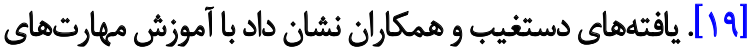

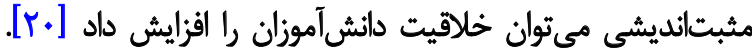

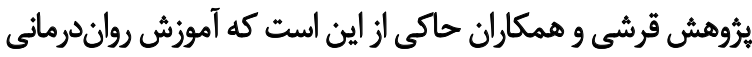

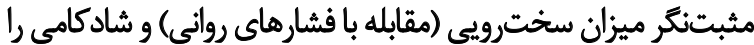

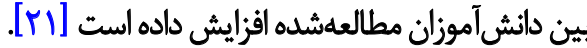

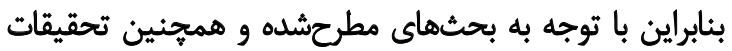

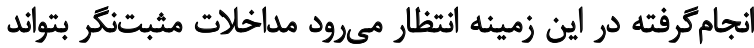

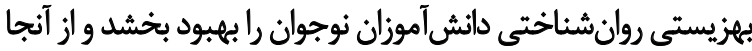

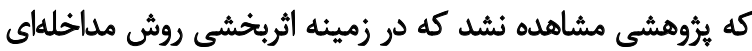

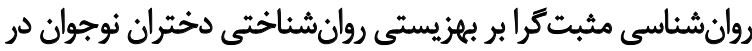

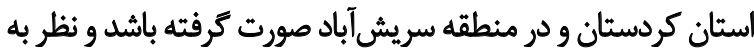

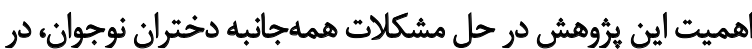

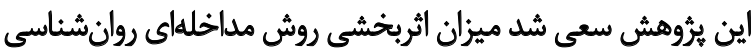

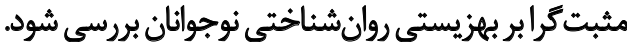

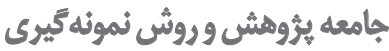

اين مطالعه شبهآزمايشى، در يكى از دبيرستانهاى دخترانه

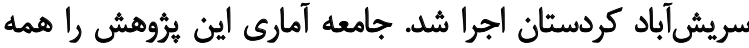

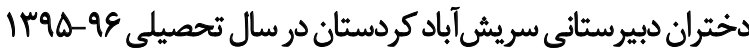

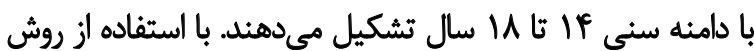
نمونه

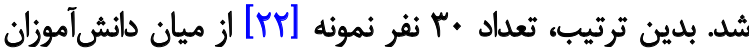

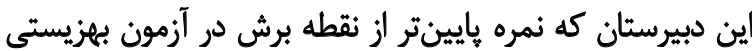

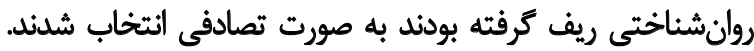

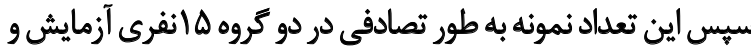

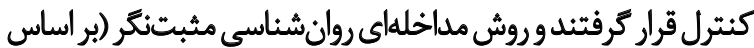

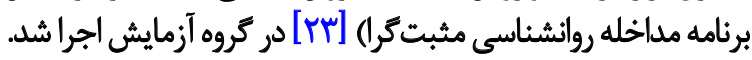

11. Froman

12. Chan

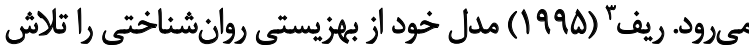

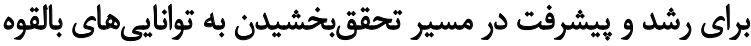

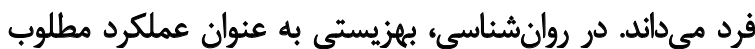
انسان قلمداد مى شود [9].

ريف (1919) شش سازه ارتباط مثبت با ديكران"، تسلط بردئ

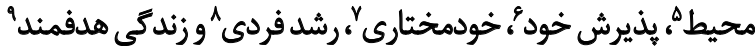

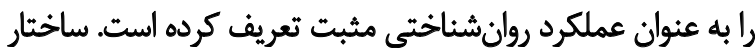

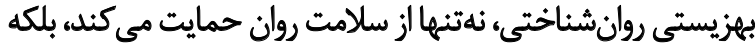

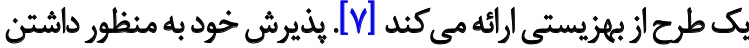

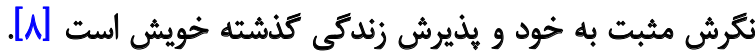

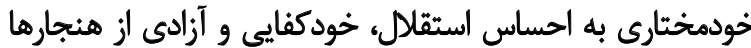

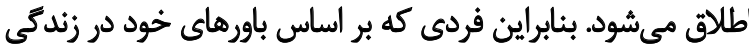

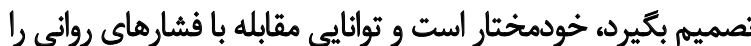

[ارد [9].

داشتن ارتباط مثبت باديكران، به معنى كيفيت رابطه خوب داشتن

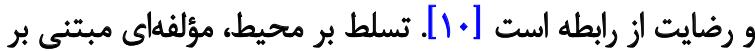

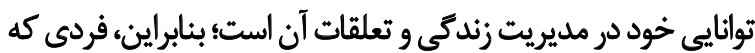

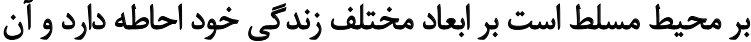

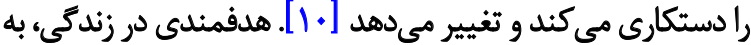

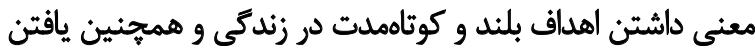

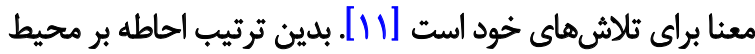

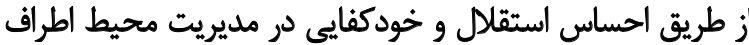

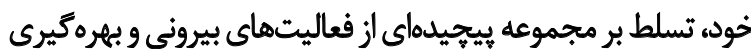

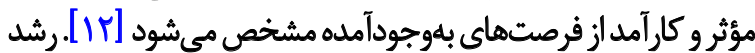

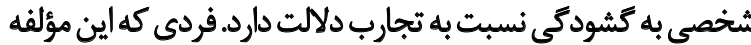

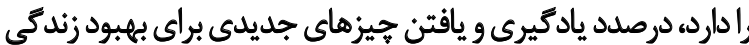

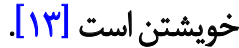

يكى از مداخلاتى كه احتمالأ بر بهزيستى روانشناختى نوجوانان

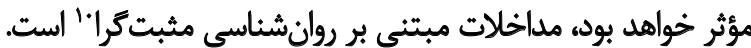

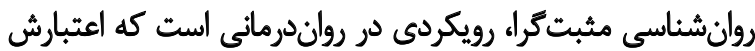

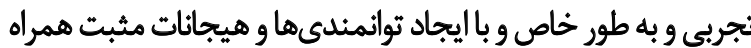

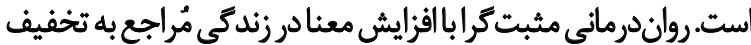

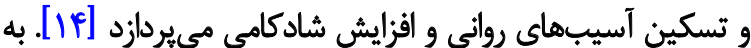

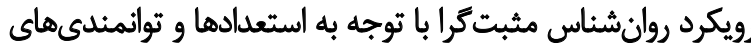

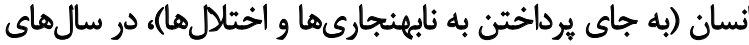

\section{Ryff \\ 4. Positive relation with other \\ 5. Environmental mastery \\ 6. Self-acceptance \\ 7. Autonomy \\ 8. Personal growth \\ 9. Purpose in life}

10. Positive Psychology Intervention 
جدول 1. خلاصه جلسات روش مداخلهاى روانشناسى مثبت

\begin{tabular}{|c|c|}
\hline محتوا & جلسه \\
\hline 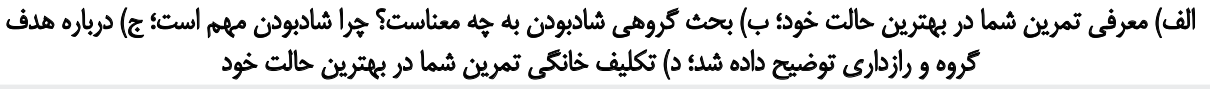 & اول (مقدمه مداخله) \\
\hline 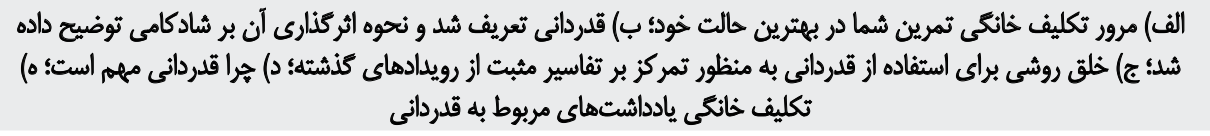 & إبراى قدردانى) \\
\hline 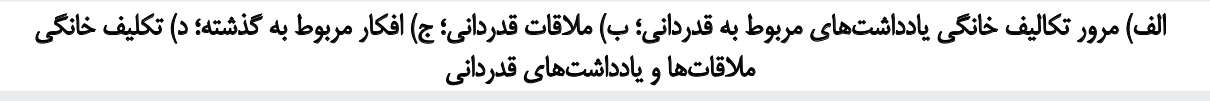 & سوم (ملاقاتهاى قدردانى)) \\
\hline 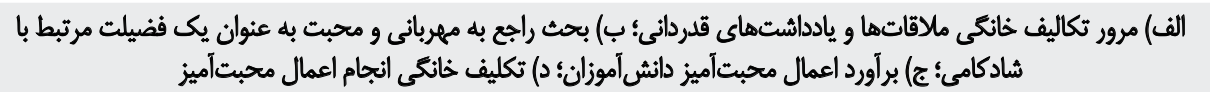 & جهارم (اعمال محبت آميز) \\
\hline 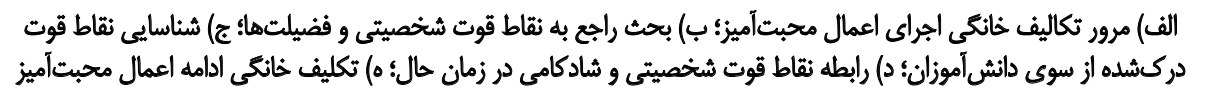 & ينجم (مقدمهاى بر نقاط قوت \\
\hline 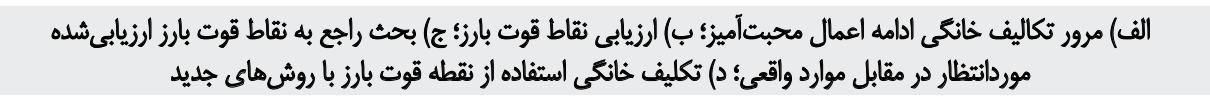 & 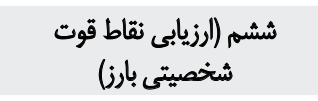 \\
\hline 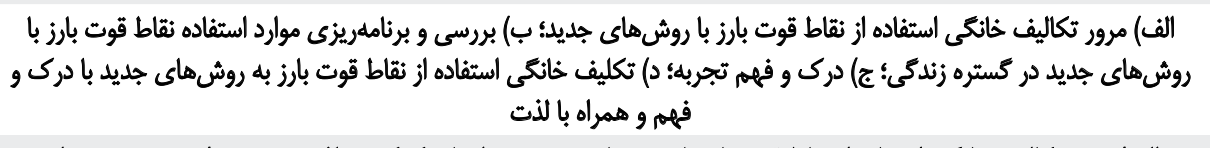 & 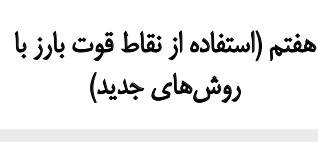 \\
\hline 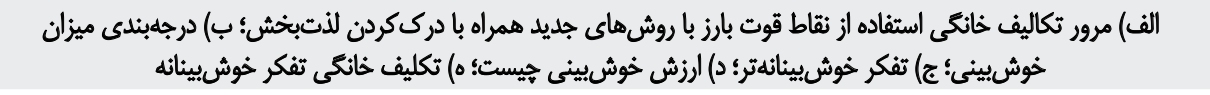 & هشتم (تفكر خوشبينانه) \\
\hline 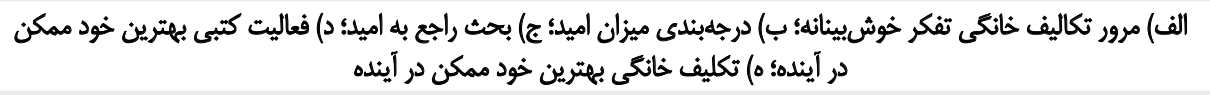 & نهم (اميد) \\
\hline 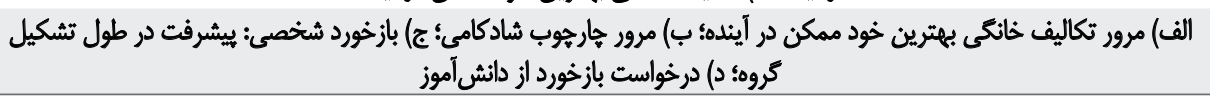 & (تمهل) \\
\hline
\end{tabular}

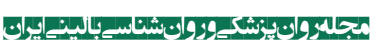

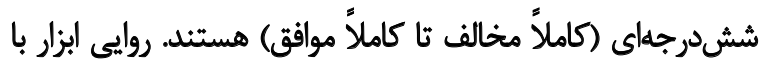

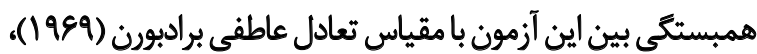

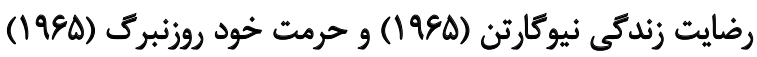

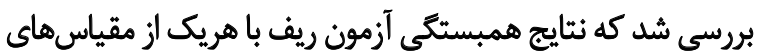

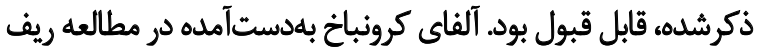

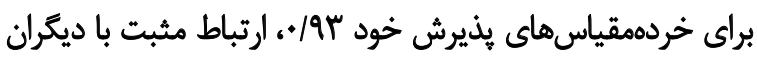

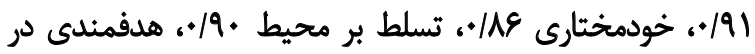

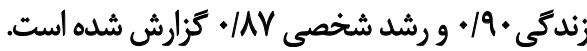

در ايران طى يروهشى كه بانمونهاي دانشجويى انجام شد، همسانى

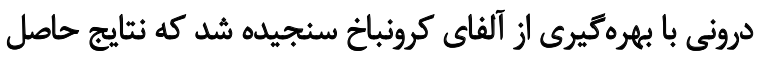

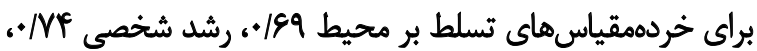

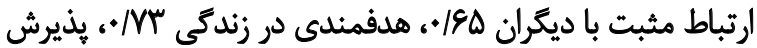

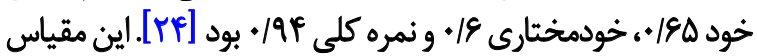

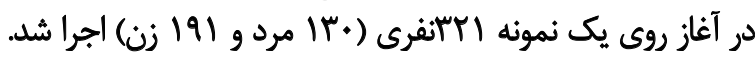

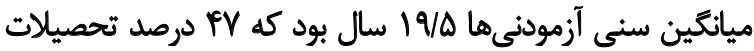

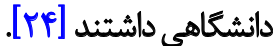

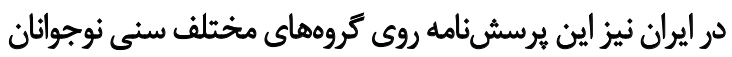

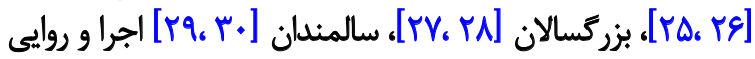

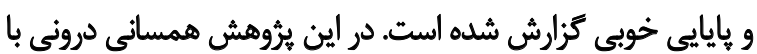

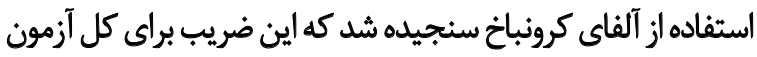

قبل از اجراى آزمون و اجراى روش مداخله مثبث إثرا، طى جلسه

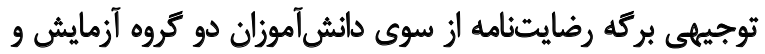

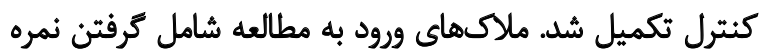

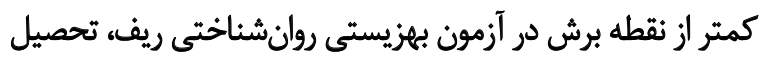

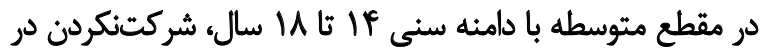

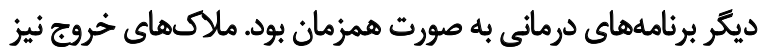

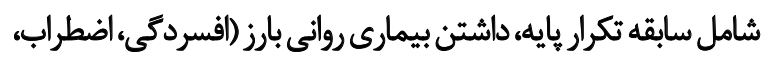

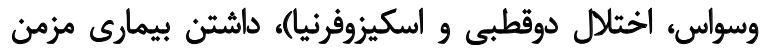

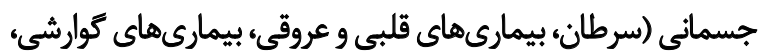

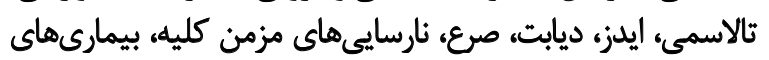

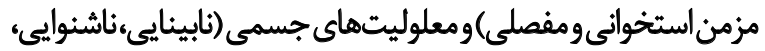

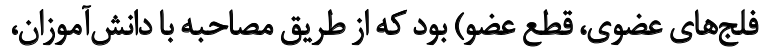

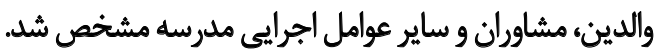

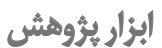

\section{مقياس بهزيستى روانشناختى ريف"}

كارول ريف (1919) اين يرسشنامه (19)

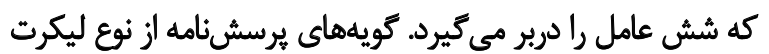

13. Ryff Psychological Well-being Scale (RPWS) 


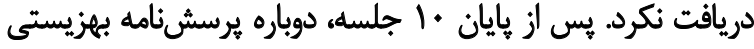

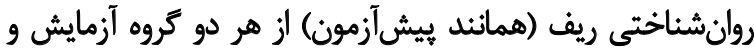

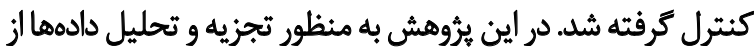

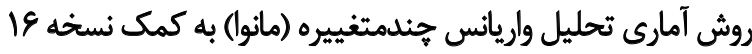

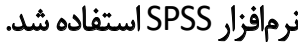

يأفتهان

ميانكين و انحراف معيار دو كروه آزمايش و كنترل در جدول

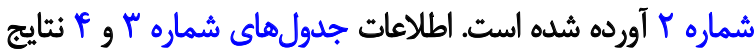

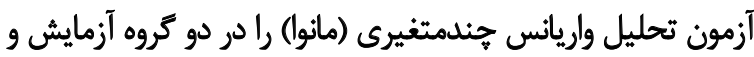

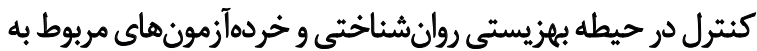

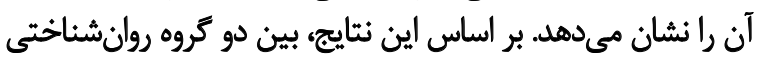

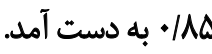

يروش مداخلهاي روانشناسي مثبت مرا مشتمل بر •1 جلسه

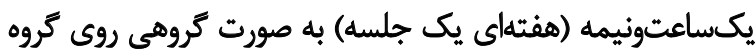

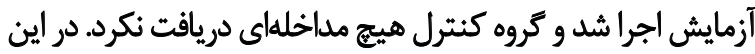

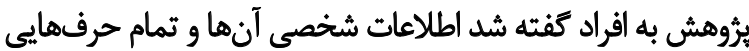

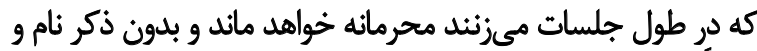

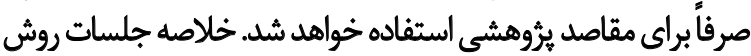

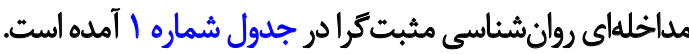

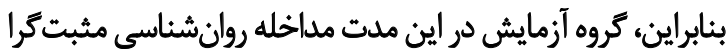

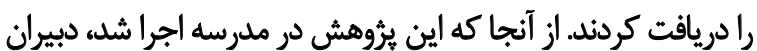

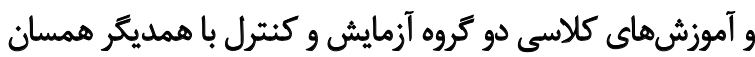

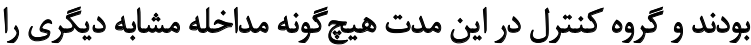

جدول r. ميانكين و انحراف معيار خردهآزمونهاى بهزيستى روانشناسى به تفكيك مراحل و كروهها

\begin{tabular}{|c|c|c|c|}
\hline ميانكين لانحراف معيار & مراحل & كروها & متغير \\
\hline 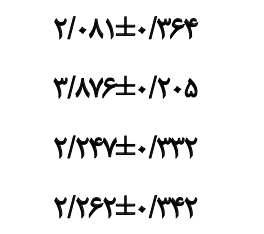 & يسيش يسآزآزمون & آزمايش & خودمختارى \\
\hline $\begin{array}{l}r / F+. \pm \cdot / q F \Delta \\
r / Q Q T \pm \cdot / r F q \\
r / q \cdot . \pm \cdot / q Q Y \\
r / \Delta) \cdot \pm \cdot / \Delta V \Delta\end{array}$ & يسيش يسآلزآزمون & آزمايش & تسلط بر هحيط \\
\hline 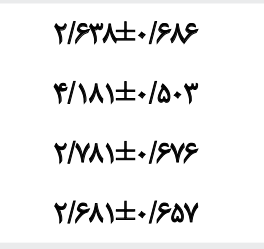 & 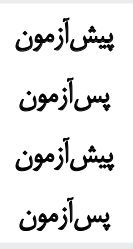 & آزمايش & رشد شخصى \\
\hline $\begin{array}{l}r / r \mu \pm . / \Delta T r \\
r / . . \pm \cdot / T F q \\
r / \Delta V I . / R q q \\
r / \Delta 1 q \pm . / \% q .\end{array}$ & 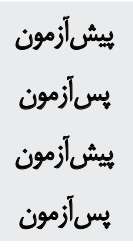 & آزمايش & روابط مثبت باديكرى \\
\hline 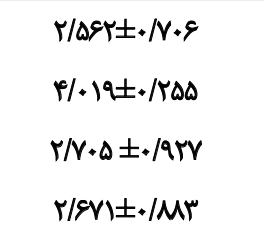 & يبي يس آزآزمون & آزمايش & هدف در زندكى \\
\hline $\begin{array}{l}r / r q \Delta \pm \cdot / M r F \\
r / Q r \Delta \pm \cdot / M P r \\
r / \Delta \Delta V \pm \cdot / \Delta \cdot r \\
r / \Delta r Y \pm \cdot / \& g r\end{array}$ & يسيش يسآزآزمون & آزمايش & ليذيرش خود \\
\hline
\end{tabular}

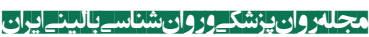


جدول ب. نتايج تحليل واريانس جندمتغيرى (مانوا) تفاوت دو گروه در روش مداخلهاي روانشناسى مثبت

\begin{tabular}{|c|c|c|c|c|c|c|}
\hline ثوان آمارى & ضريب اتا & سطح معنادارى & ضريب F & درجه آزادى & لامبداى ويلكس & مثابع \\
\hline $1 \ldots$ &.$/ 949$ & .10 .1 & $r \Delta r / I T V$ & $r$ & $\cdot|+\Delta|$ & Sرو \\
\hline
\end{tabular}

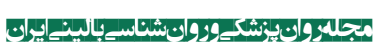

جدول P. خلاصه تحليل واريانس جندمتغيرى (مانوا) تفاوت برحسب عضويت كروهي در خردهآزمونهاي بهزيستى روانشناختى

\begin{tabular}{|c|c|c|c|c|c|c|c|}
\hline توان آماري & ضريب اتا & معنادارى سطع & ضريب F & مجذيانئين & أزاديه & مجموع مجذورات & شاخص أمارى مثغيرها \\
\hline 1 &.$/ 14 u$ & $+1+\infty 1$ & Tra/AهA & 19/AFF & 1 & 19/DFF & خودمختارى \\
\hline 1 &.$/ M F T$ & $+1+\infty$ & $\Lambda \cdot|\Delta \Lambda|$ & $|Q / A T|$ & 1 & |Q/ATI & تسلط بر محيط \\
\hline 1 &.$/ 941$ & $+1+.1$ & Fq/TrY & IF/AVA & 1 & IF/AVA & رشد شخصى \\
\hline 1 & - AAfE & .1 .01 & $\operatorname{lar} / A \cdot f$ & iepleq & 1 & $18 / p$ eq & روابط هثبت باديكرى \\
\hline 1 & . /Ora & .10 .1 & $r T / r f$. & $|H / E T|$ & 1 & $|r / g T|$ & هدف نر زندكى \\
\hline 1 & $\cdot / A \cdot r$ & $.1 . .1$ & $111 / \Delta P^{\prime}$ & $10 / \cdot \cdot r$ & 1 & $10 / \cdot \cdot r$ & يذيرش خود \\
\hline
\end{tabular}

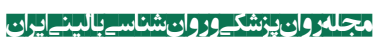

فشارهاى روانى) دانش آموزان مطالعهشده مؤثر است و آن را افزايش

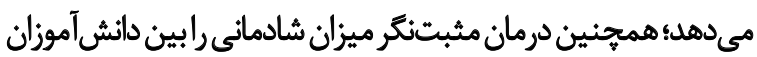

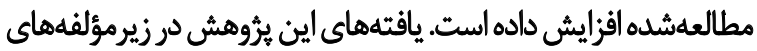

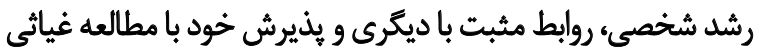

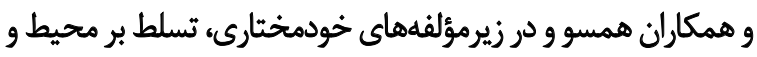

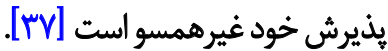

مداخله مثبتنكر كروهى باعث افزايش هيجانهاى مثبت،

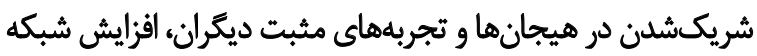

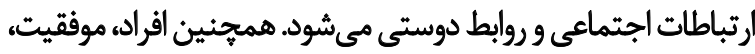

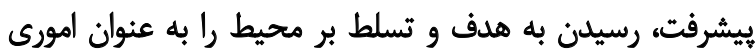

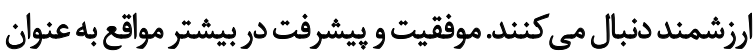

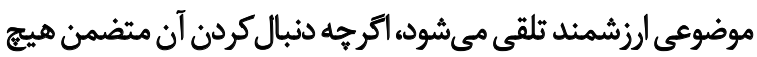

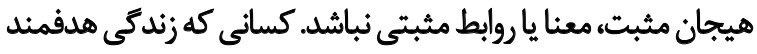

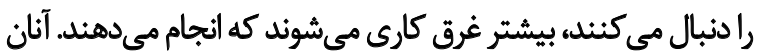

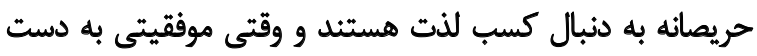

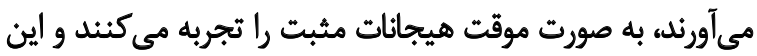

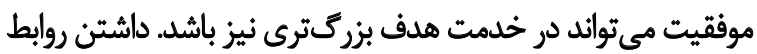

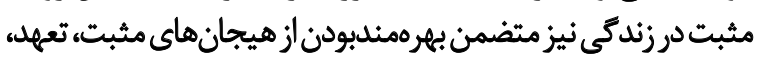

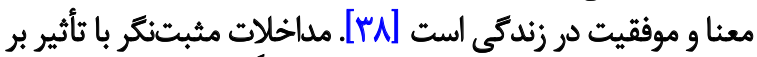

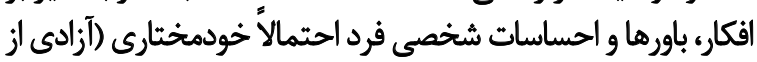

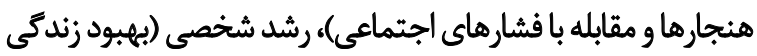

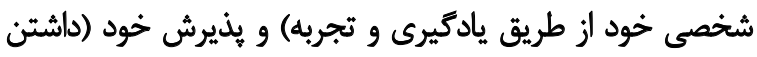

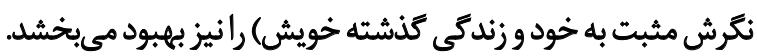

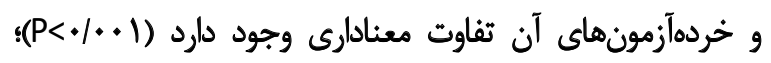

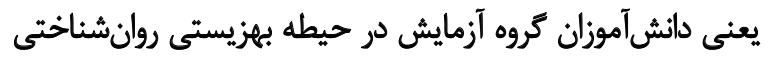

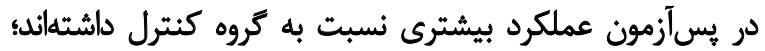

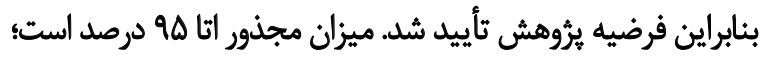

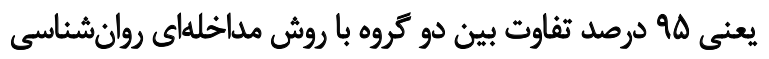

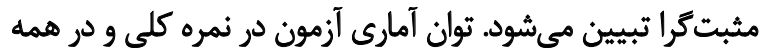
خردهمقياسها ا است كه دلالت بر كفايت حجمي نمونه دارد.

\section{ث}

در اين يثروهش اثربخشى روش مداخلهاى روانشناسى مثبت مرا بر بهزيستى روانشناختى نوجوانان بررسى شد. تجزيه و تحليل دانيل دادهها

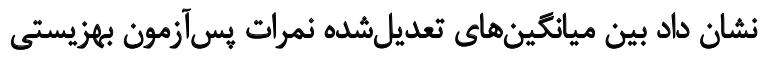

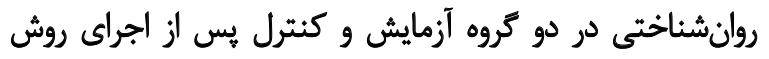

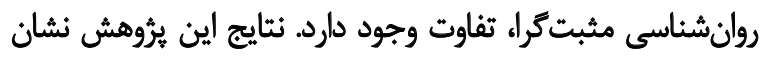

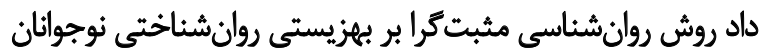

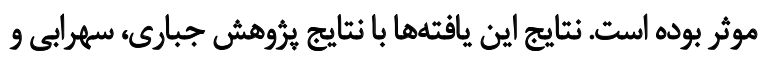

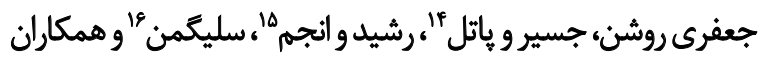

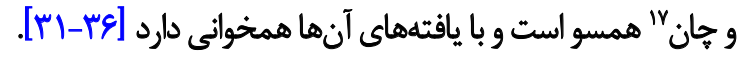
نتايج يُوهش قرشى و همكاران [Yl] نشان داد رواندرمانى

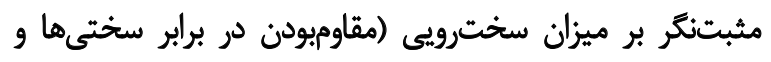

14. Jaser \& Patel

15. Anjum

16. Seligman

17. Chan 


$$
\text { هامى مالى }
$$

مفهومسازى، كردآورى، تحليل و نوشتن: بيان نسائي مقدم؛ كمك

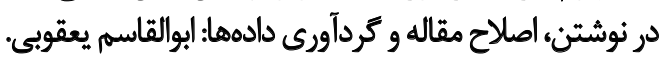

$$
\text { تقارض مناقع }
$$

بنابر اظهار نويسندكان، اين مقاله تعارض منافع ندارد.

\section{تئيجليرى}

با وجود اصلاحات در آموزش و يرورش و مسائل آموزشى در اواخر

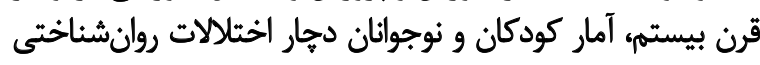

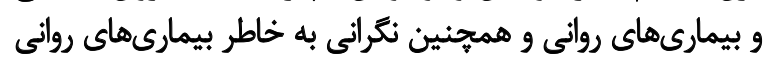

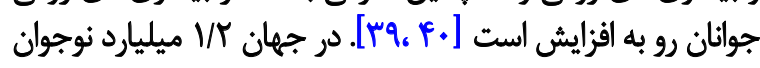

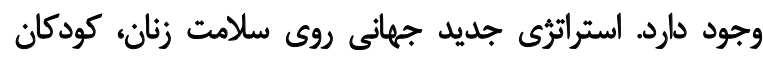

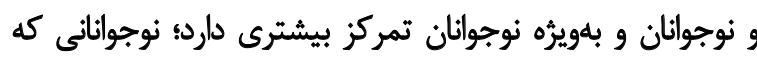

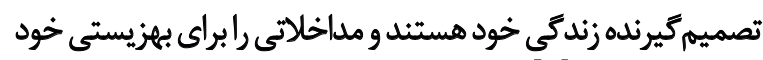

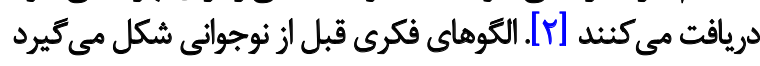

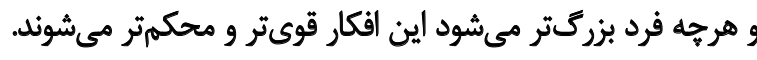

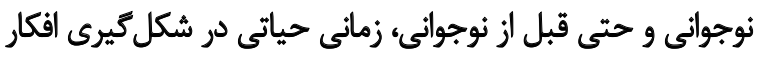

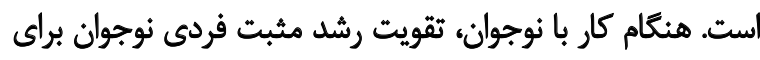

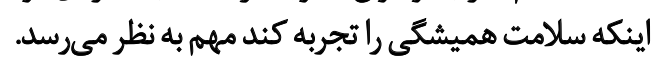

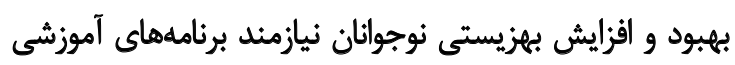

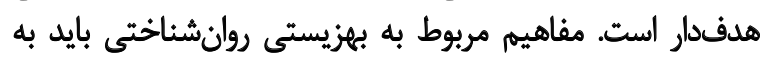

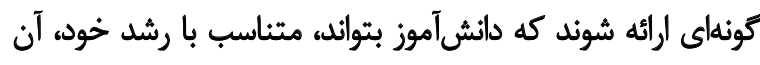

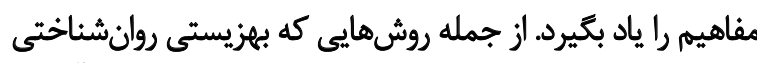

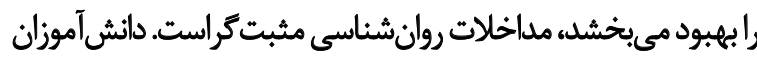

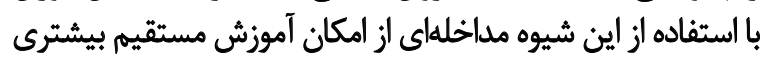
بهرهمندمىشوند.

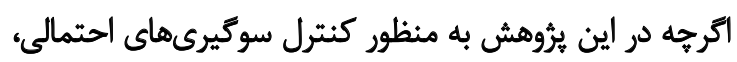

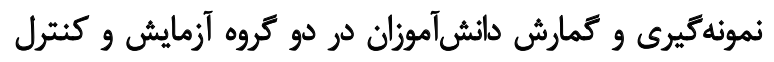

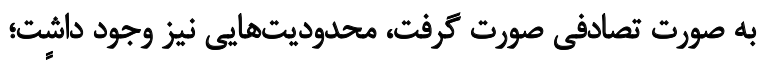

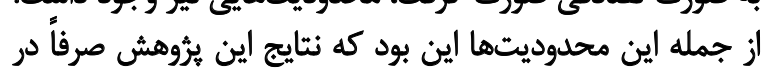

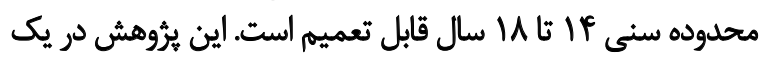

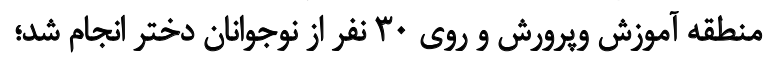

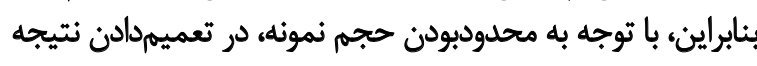

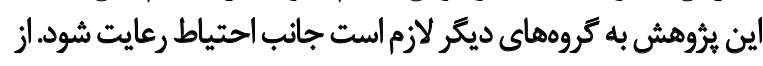

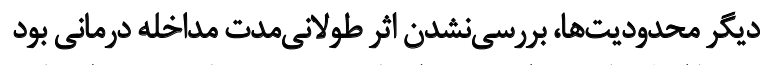

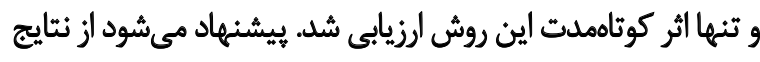

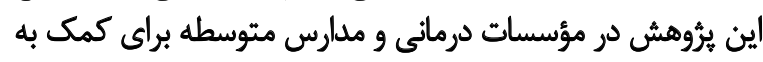

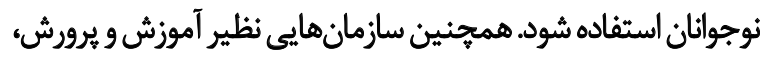

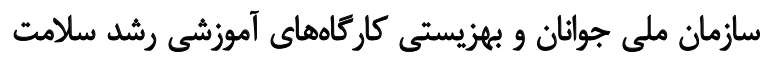

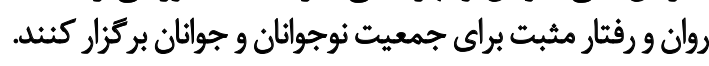

\section{مالاحظات اخلاقي}

\section{يميروى أز اصول اخغاق بثوهش}

همه اصول اخلاقي در اين مقاله رعايت شده است. شركت كنيندكان

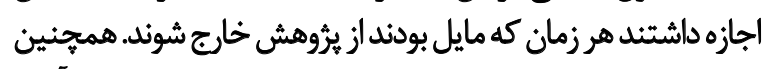

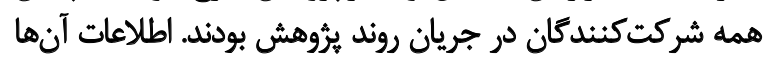
محرمانه نكه داشته شد شكن. 


\section{References}

[1] Pompili M, Masocco M, Innamorati M. Suicide among Italian adolescents: 1970-2002. European Child and Adolescent Psychiatry. 2009; 18(9):525-33. [DOI:10.1007/s00787-009-0007-x]

[2] Reavley NJ, Sawyer SM. Improving the methodological quality of research in adolescent well-being. New York: UNICEF; 2017.

[3] Rice F. Human growth: The psychology of birth to death [M Foroughan, Persian trans.]. Tehran: Arjmand; 2017.

[4] Fergusson DM, Woodward LJ. Mental health, educational and social role outcomes of adolescents with depression. JAMA Psychiatry. 2012; 59(3):31-225.

[5] Schuster MA, Eastman KL, Fielding JE, Rotheram-Borus MJ, Breslow L, Franzoi LL, et al. Promoting adolescent health: Worksite-based intervention with parents of adolescents. Journal of Public Health Management \& Practice. 2001; 7(2):41-52. [DOI:10.1097/00124784-200107020-00007]

[6] Nel L. The psychofertological experiences of master s degree stuents in professional psychology programms: An interpretative phenomenological analysis [PhD. dissertation]. Bloemfontein: University of The Free State; 2011.

[7] Garcia D, Archer T, Moradi S, Andersson-Arntén AC. Exercise frequency, high activation positive affect, and psychological wellbeing: Beyond age, gender, and occupation. Psychology. 2012; 3(4):328-36. [DOI:10.4236/psych.2012.34047]

[8] Hauser RM, Springer KW, Pudrovska T. Temporal structures of psychological well-being continuing change. Paper presented at: The 2005 Meetings of the Gerontological Society of America Orlando. 20 November 2005; Orlando, Florida, USA.

[9] RyffCD, Singer B. The contours of positivehuman health. Psychological Inquiry. 1998; 9(1):1-28. [DOI:10.1207/s15327965pli0901_1]

[10] Keyes CL. The mental health continuum: From languishing to flourishing in life. Journal of Health and Social Behavior. 2002 43(2):207-22. [DOI:10.2307/3090197]

[11] Ryff CD. Happiness is everything, or is it? Explorations on the meaning of psychological well-being. Journal of Personality and Social Psychology. 1989; 57(6):1069-81. [DOI:10.1037/00223514.57.6.1069]

[12] Shokri A, Kadivar P, Farzad A, Daneshvarpour Z, Dastjerdi R, Autumn M. [Investigating the factor structure of persian versions 3, 9 and 14 of the question of reef psychological well-being scales in students (Persian)]. Iranian Journal of Psychiatry and Clinical Psychology. 2009; 14(2):152-6.

[13] Lindfors P, Berntsson B, Lundberg U. Factor structure of Ryff's psychological well being scales in Swedish female and male whitecollar workers. Personality and Individual Differences. 2006; 40(6):113-22. [DOI:10.1016/j.paid.2005.10.016]

[14] Rashid T. Positive psychotherapy. In Lopez SJ, editor. Positive Psychology: Exploring the Best in People. Westport, Connecticut: Greenwood Publishing Company; 2008.

[15] Easterlin RA. Income and happiness: Towards a unified theory. The Economic Journal. 2001; 111(473):465-84. [DOI: 10.1111/14680297.00646]
[16] Froman L. Positive psychology in the workplace. Journal of Adult Development. 2010; 17(2):59-69. [DOI:10.1007/s10804009-9080-0]

[17] Chan FM. Developing information literacy in Malaysian Smart schools: resource-based learning as a tool to prepare todays students for tomorrow, s society. Paper presented at: Annual Conference of the International Association of School Librarianship. 5-9 August 2002; Petaling Jaya, Malaysia.

[18] Brand M, Young KS, Laier C, Wölfling K, Potenza MN. Integrating psychological and neurobiological considerations regarding the development and maintenance of specific Internet-use disorders: An Interaction of Person-Affect-Cognition-Execution (I-PACE) model. Neuroscience \& Biobehavioral Reviews. 2016; 71:252-66. [DOI:10.1016/j.neubiorev.2016.08.033]

[19] O'Connell BH, O'Shea D, Gallagher S. Enhancing social relationships through positive psychology activities: A randomised controlled trial. The Journal of Positive Psychology. 2016; 11(2):149-62. [DOI:10.1080/17439760.2015.1037860]

[20] Dasgheib SM, Alizadeh H, Farrahi NA. [The effect of positive thinking skills on the creativity of first high school girl girls (Persian)]. Journal of Criticism and Innovation in the Humanities. 2012; 1(4):1-17

[21] Ghorashi S, Bakhtiari A, Fard F. [The consideration of positive perspective psychotherapy on hard work and happiness of female students of high school city of Marand 2015 (Persian)]. Journal of Counseling and Psychotherapy. 2016; 4(15):60-75.

[22] Delavar A. [Research methods in psychology and educational sciences (Persian)]. Tehran: Payame Noor University; 1992.

[23] Savage AS. Increasing adolescents' subjective well-being: Effects of a positive psychology intervention in comparison to the effects of therapeutic alliance, youth factors and expectancy for change [PhD. dissertation]. Florida: University of South Florida; 2011.

[24] Ryff CD. Psychological well-being in adult life. Current Directions in Psychological Science. 1995; 4(4):99-104. [DOI:10.1111/1467-8721.ep10772395]

[25] Jabbari M, Shahidi S, Mootabi F. [Effectiveness of group intervention based on positive psychology in reducing symptoms of depression and anxiety and increasing life satisfaction in adolescent girls (Persian)]. Iranian Journal of Psychiatry and Clinical Psychology. 2015; 20(4):296-87.

[26] Moghanibashi E, Asgharipour N. [Effectiveness of coping skills training on depression and psychological well-being among girl adolescents (Persian)]. Horizon. 2015; 21(4):117-22.

[27] Bayani AA, Mohammad Koochekya A, Bayani A. [Reliability and validity of Ryff's Psychological Well-being Scales (Persian)] Iranian Journal of Psychiatry and Clinical Psychology. 2008; 14(2):146-51.

[28] Shokri O, Kadivar P, Farzad V, Daneshvarpour Z, Dastjerdi R, Paeezi M. [A study of factor structure of 3, 9 and 14-item Persian versions of Ryff's Scales Psychological Well-being in university students (Persian)]. Iranian Journal of Psychiatry and Clinical Psychology. 2008; 14(2):152-61.

[29] Sohrabi M, Abedanzade R, Shetab Boushehri N, Parsaei S, Jahanbakhsh $\mathrm{H}$. [The relationship between psychological well-being and mental toughness among elders: Mediator role of physical activity (Persian)]. Iranian Journal of Ageing. 2017; 11(4):538-49. 
[30] Moatamedy A, Borjali A, Sadeqpur M. [Prediction of psychological well-being of the elderly based on the power of stress management and social support (Persian)]. Iranian Journal of Ageing. 2018; 13(1):98-109.

[31] Jabbari M, Shahidi S, Motabi F. [The effectiveness of positive intervention on reducing inefficient attitudes and increasing the happiness of adolescent girls (Persian)]. Journal of Clinical Psychology. 2008; 6(2):65-74. [DOI:10.22075/JCP.2017.2164]

[32] Sohrabi F, Jafari Roshan F. [Effectiveness of positive group psychotherapy on resiliency, happiness and general health on women with a substance dependence spouses (Persian)]. Positive Psychology Research. 2014; 2(1):31-45.

[33] Jaser S, Patel N. Development of a positive psychology intervention to improve adherence in adolescents with type 1 diabete. Journal of Pediatric Health Care. 2014; 28(6):478-85. [DOI: 10.1016/j.pedhc.2014.02.008] [PMCID] [PMID]

[34] Rashid T, Anjum, A. Positive psychotherapy for young adults and children. In Abela JRZ, Hankin BL, editors. Handbook of Depression in Children and Adolescents. New York: Guilford Press; 2008.

[35] Seligman ME, Rashid T, Parks AC. Positive psychotherapy. American Psychologist. 2006; 61(8):774-88. [DOI:10.1037/0003066X.61.8.774]

[36] Chan H. Mental health care for children. Childhood Disorders Journals. 2017; 3:13-4. [DOI:10.4172/2472-1786.100051]

[37] Ghiasi SE, Tabatabaee TS, Nasri M. [Effect of optimism training on emotion regulation and psychological well-being in undergraduate students of University of Birjand, in 2014-2015 (Persian)]. Pajouhan Scientific Journal. 2016; 14(4):27-37.

[38] Seligman ME, Godario R. Positive programs and their's effects on disabled children. Journal of Disability. 2017; 56:67-79.

[39] Oades LG, Robinson P, Green S. Positive education: Creating flourishing students, staff and schools. Australian Psychological Society. 2011;33(2):16-7.

[40] Woods R, Pooley JA. A review of intervention programs that assist the transition for adolescence into high school and the prevention of mental health problems. International Journal of Child and Adolescent Health. 2015; 8(2):97-108. 
This Page Intentionally Left Blank 